\title{
The potential link between PML NBs and ICP0 in regulating lytic and latent infection of HSV-1
}

\author{
Shuai Wang, Jing Long, Chun-fu Zheng ${ }^{\bowtie}$ \\ State Key Laboratory of Virology, Wuhan Institute of Virology, Chinese Academy of Sciences, Wuhan 430071, China \\ $\triangle$ Correspondence: zhengcf@wh.iov.cn \\ Received December 7, 2011 Accepted January 19, 2012
}

\begin{abstract}
Herpes simplex virus type 1 (HSV-1) is a common human pathogen causing cold sores and even more serious diseases. It can establish a latent stage in sensory ganglia after primary epithelial infections, and reactivate in response to stress or sunlight. Previous studies have demonstrated that viral immediate-early protein ICPO plays a key role in regulating the balance between lytic and latent infection. Recently, It has been determined that promyelocytic leukemia (PML) nuclear bodies (NBs), small nuclear sub-structures, contribute to the repression of HSV-1 infection in the absence of functional ICPO. In this review, we discuss the fundamentals of the interaction between ICPO and PML NBs, suggesting a potential link between PML NBs and ICPO in regulating lytic and latent infection of HSV-1.
\end{abstract}

KEYWORDS herpes simplex virus type 1 (HSV-1), reactivation, ICP0, promyelocytic leukemia (PML), speckled protein of $100 \mathrm{kDa}(\mathrm{Sp} 100)$

\section{INTRODUCTION}

Herpes simplex virus type 1 (HSV-1), the archetypal member of the alphaherpes virus subfamily, is a large, nuclear-replicating, dsDNA virus. HSV-1 is a human neurotropic virus and infects mucous membranes causing cold sores. Following primary infection in epithelial cells, HSV-1 establishes lifelong latent infection in sensory neurons of the trigeminal ganglia by getting access to nerve terminals, leading to periodic reactivation and lytic replication at the site of original infection. Latency is such a status when viral genome adopts a non-linear configuration and is transcriptionally repressed with the exception of a region encoding the latency-associated transcripts (LATs). Provoked by stress stimuli that act on the neuron, the latent virus reactivates and switches to a productive viral replication, allowing the virus to spread. Although there are large numbers of literatures related to HSV-1 latency and reactivation, the mechanisms by which viral genomes are maintained in a repressed state during latency and the processes involved in reactivation from latency are still not fully understood (Mitchell et al., 2003; Shimomura, 2008).

Previous studies have demonstrated that viral immediate-early protein ICPO, a promiscuous activator of gene expression, is responsible for efficient entry into lytic cycle and can induce reactivation of latent or quiescent viral genomes, which have led to the suggestion that it plays a key role in regulating the balance between lytic and latent HSV-1 infection (Everett, 2000; Preston, 2000; Halford et al., 2001; Everett et al., 2004a; Hagglund and Roizman, 2004).

It is proposed that in the absence of functional ICPO, a promyelocytic leukemia (PML) nuclear bodies (NBs) related cellular repression mechanism silences viral transcription. ICPO seems to counteract this process by stimulating the degradation of a number of cellular proteins via the ubiquitin-proteasome pathway (Table 1) (Boutell et al., 2002;

Table 1 Client proteins degraded by ICPO

\begin{tabular}{ll}
\hline Protein & Reference \\
\hline PML & Chelbi-Alix and de Thé, 1999 \\
Sp100 & Chelbi-Alix and de Thé, 1999 \\
ATRX & Everett et al., 2007 \\
hDaxx & Everett et al., 2007 \\
E2FBP1 & Fukuyo et al., 2011 \\
Centromeric proteins CENP-A & Lomonte et al., 2001 \\
CENP-B & Lomonte et al., 2007 \\
CENP-C & Everett et al., 1999 \\
Catalytic subunit of DNA-PK & Lees-Miller et al., 1996 \\
\hline
\end{tabular}


Everett et al., 2006; Everett et al., 2008a).

\section{LYTIC AND LATENT INFECTION OF HSV-1}

Evolutionary pressures by viral infection ensure that the host develops defense systems to eliminate virus, while viruses have adopted many strategies to outmaneuvre the host's immune and other anti-viral systems while maintaining their long term survival potential. HSV-1 has successfully developed a particularly exquisite form of accommodation within the host. Animal experiments revealed that the virus gains access to the termini of sensory neurons during primary infection in epithelial cells, and is transported by retrograde axonal transport to the sensory ganglia, where it replicates briefly and then establishes a latent infection (Mitchell et al., 2003; Shimomura, 2008) .

Lytic HSV-1 infection involves abundant transcription from the entire viral genome in a regulated cascade of immediate-early (IE), early, and late gene products (Lehman and Boehmer, 1999). The IE gene products regulate the expression of later classes of viral genes. During latent cycle, the transcription of HSV-1 genome is repressed, and only LATs, which derive from a single locus that lies countersense to the IE gene encoding ICPO, are expressed in readily detectable amounts (Wagner and Bloom, 1997; Preston, 2000; Efstathiou and Preston, 2005). HSV-1 thus establishes a life-long infection in a form that is not accessible to anti-viral defense. Periodically, episodes of lytic infection occur as a result of reactivation.

HSV-1 latency is therefore a key component of its life cycle, and as such the underlying mechanisms have been a subject of considerable interest. In particular, the regulation of viral gene expression during various stages of lytic and latent infection has been a very active and productive field of research (Wagner and Bloom, 1997).

\section{ICP0, a key activator of HSV-1 gene expression}

During successional steps of HSV-1 lytic cycle gene expression, the IE genes are the first to be transcribed, and their products are required for the activation of later classes of promoters. The main viral transactivators required for the expression of the HSV-1 genes are the IE proteins ICPO, ICP4 and the late protein VP16 (Table 2).

VP16, a component of the HSV tegument which is released into the cell following fusion of viral envelope, binds to cellular factors HCF and Oct-1, initiating the whole genetic program by activating the expression of the IE genes (Mahajan et al., 2002; Wysocka and Herr, 2003).

ICP4, a factor exerting its transcriptional activity by binding specifically or nonspecifically to DNA (Smith et al., 1993), is absolutely required for the transactivation of the HSV-1 early and late genes (Watson and Clements, 1980; DeLuca et al., 1985).

ICP0, which appears to act upstream of other IE proteins, is a crucial regulator of the three classes of HSV genes and has also been shown to be able to transactivate several other heterologous promoters in transfection reporter assays. It had been established earlier that ICP0 and ICP4 produce a synergistic transactivation of those promoters, greatly exceeding that in the presence of either protein alone. ICPO reactivates quiescent viral genomes and stimulates initiation of Iytic infection by transactivating a broad range of viral and cellular promoters, suggesting crucial roles of ICPO in different stages of HSV-1 infection (Preston and Nicholl, 1997; Samaniego et al., 1998).

Table 2 Expression time course and function of ICPO

\begin{tabular}{|c|c|c|c|}
\hline & Expression time course & Function & Reference \\
\hline \multirow{7}{*}{ ICPO } & \multirow{7}{*}{$\begin{array}{l}\text { Immediate } \\
\text { early }\end{array}$} & $\begin{array}{l}\text { Activate later classes of promoters and enhance viral } \\
\text { transcription }\end{array}$ & Cai and Schaffer, 1992 \\
\hline & & Required for reactivation from latency & Halford and Schaffer, 2001 \\
\hline & & $\begin{array}{l}\text { E3 ubiquitin ligase activity, and degrade cellular proteins } \\
\text { by ubiquitin-proteasome pathway }\end{array}$ & Boutell et al., 2002 \\
\hline & & Disrupt PML NBs & Maul et al., 1993 \\
\hline & & Relocate class II histone deacetylase & Lomonte et al., 2004 \\
\hline & & $\begin{array}{l}\text { Prevent the accumulation of histones, repress histone } \\
\text { modifications }\end{array}$ & Ferenczy and DeLuca, 2011 \\
\hline & & Counteract type I interferon (IFN) response & Mossman and Smiley, 2002 \\
\hline \multirow{3}{*}{ ICP4 } & \multirow{3}{*}{$\begin{array}{l}\text { Immediate } \\
\text { early }\end{array}$} & Transactivation & Watson and Clements, 1980 \\
\hline & & Transcription repression & Paterson and Everett, 1988 \\
\hline & & Initiate viral DNA replication & Aslani et al., 2000 \\
\hline \multirow[t]{2}{*}{ VP16 } & \multirow[t]{2}{*}{ Late } & $\begin{array}{l}\text { Recruit general transcription factors, RNA polymerase II, } \\
\text { histone acetyltransferases (HATs), and ATP dependent } \\
\text { chromatin remodeling complexes to IE promoters, stimu- } \\
\text { late immediate-early gene expression }\end{array}$ & $\begin{array}{l}\text { Harris and Preston, 1991; Xiao et al., 1994; } \\
\text { Klemm et al., 1995; Memedula and Bel- } \\
\text { mont, 2003; Mittler et al., 2003; Herrera and } \\
\text { Triezenberg, 2004; von Einem et al., } 2006\end{array}$ \\
\hline & & Virions assembly & von Einem et al., 2006 \\
\hline
\end{tabular}


The function of ICPO has been extensively studied, and an increasingly detailed picture of its interactions with cellular proteins and its biochemical functions is emerging (Kawaguchi et al., 1997a, 1997b; Everett et al., 1998; Everett et al., 1997; Parkinson and Everett, 2000; Kawaguchi et al., 2001; Van Sant et al., 2001; Boutell et al., 2002; Boutell and Everett, 2003; Gu and Roizman, 2003; Jackson and DeLuca, 2003). Direct transcriptional activators either bind to specific response elements in target promoters or interact with host transcription factors that form part of the basal transcriptional machinery or associated activator complexes. Whereas ICPO itself does not bind DNA directly, it seems that ICPO acts as a transactivator of viral genome and a specific subset of cellular genes through interaction with some cellular proteins. Several studies reported that ICPO interacts with various cellular factors, including cyclin D3, elongation factor EF-1 $\sigma$, transcription factor BMAL1, ubiquitin-specific protease HAUSP (Everett et al., 1997; Kawaguchi et al., 1997a, 1997b) and also specific nuclear structures known as PML NBs (Gu and Roizman, 2003; Lukashchuk and Everett, 2010).

Furthermore, one of the key roles of ICPO relates to its expression of two E3 ubiquitin ligase activities located in exons 2 and 3 (Van Sant et al., 2001; Boutell et al., 2002; Hagglund and Roizman, 2002; Hagglund et al., 2002). The ubiquitin ligase activity present in exon 2 is associated with a RING finger domain and is responsible for the proteasome-mediated degradation of cellular proteins, including major proteins associated with PML NBs such as PML and speckled protein of $100 \mathrm{kDa}$ (Sp100), centromeric proteins CENP-A and CENP-C, and the catalytic subunit of DNAdependent protein kinase (Everett et al., 1998a; Everett et al., 1999a; Lomonte et al., 2001; Gu and Roizman, 2003). The second ubiquitin ligase activity identified in exon 3 is responsible for the degradation of the E2 ubiquitin-conjugating enzyme cdc34 (Van Sant et al., 2001; Hagglund and Roizman, 2003). The present hypothesis to explain the wide transactivating or derepressing activities of ICPO, as well as its implication in the establishment of lytic replication, is the ability of ICPO to alter the higher-order structure of chromatin by targeting a repressive factor for degradation (Everett, 2000) (Table 1).

\section{PML NBs, small nuclear sub-structures with multifunction}

PML NBs, also known as nuclear dot 10 (ND10) or PODs (PML oncogenic domains), are small nuclear sub-structures with a striking punctate appearance. The size of PML NBs is between 0.2 and $1 \mu \mathrm{m}$ and its quantity is from $2-3$ to 30 per cell, depending on the cell type and status. PML NBs are dynamic macromolecular inclusions of cellular proteins that form within the interchromosomal space in the nucleus (Sternsdorf et al., 1997a; Everett et al., 1999b; Dellaire et al., 2006), functioning in oncogenesis, the DNA damage re- sponse (Dellaire and Bazett-Jones, 2004), the stress response, apoptosis (Bernardi and Pandolfi, 2003; Gresko et al., 2009), senescence (Bischof et al., 2002), the ubiquitin pathway (Antón et al., 1999; Lallemand-Breitenbach et al., 2001), various genetic disorders, viral infection and the interferon (IFN) response (Regad et al., 2001).

An increasing list of cellular proteins has been proven to accumulate at PML NBs; those proteins can be divided into two categories, one is permanent components, such as PML, Sp100, the death domain-associated protein (Daxx), small ubiquitin like modifier (SUMO), and the bloom syndrome helicase (BLM), and the other category is proteins that present in PML NBs only under specific circumstances (e.g. DNA repair machinery) or during overexpression (e.g. breast cancer protein BRCA1) (Negorev and Maul, 2001; Tavalai et al., 2008).

PML (also known as TRIM19), a tripartite motif family protein, is the key component of PML NBs and required for assembly of these structures (Ishov et al., 1999; Zhong et al., 2000). Posttranslational modification of PML by covalent conjugation to SUMO at K65, 160, 490, named SUMOylation, is required for proper formation of PML NBs and recruitment of PML NB-associated proteins (Sternsdorf et al., 1997b; Kamitani et al., 1998a, 1998b; Müller et al., 1998; Duprez et al., 1999; Ishov et al., 1999; Zhong et al., 2000; Seeler and Dejean, 2001; Shen et al., 2006). Sp100, another permanent component of PML NBs, is also found to be conjugated to SUMO; however, SUMOylation of Sp100 is not necessary for its localization to PML NBs (Sternsdorf et al., 1997b; Sternsdorf et al., 1999).

\section{PML NBs confer intrinsic resistance to viral infection}

PML NBs display intrinsic antiviral properties, targeting both DNA viruses and cytoplasmic replicating RNA viruses (Regad et al., 2001; McNally et al., 2008; Tavalai et al., 2008). Unlike cytokine-mediated responses, intrinsic antiviral resistance involves the actions of pre-existing cellular proteins to repress viral transcription (Saffert and Kalejta, 2008; Tavalai et al., 2008). PML NBs have been demonstrated to repress the replication of HSV-1 shortly after virus entry by mechanisms that limit early viral gene transcription, although the process is counteracted by ICPO (Everett et al., 2006; Everett et al., 2007). Early studies also show that exogenous expression of $\mathrm{PML}$ isoform $\mathrm{VI}$ causes significant reduction of adenovirus (Doucas et al., 1996) and human cytomegalovirus (HCMV) infection (Ahn and Hayward, 2000), while depleting PML enhances varicella-zoster virus (VZV) and HCMV replication (Tavalai et al., 2006; Tavalai et al., 2008; Kyratsous and Silverstein, 2009). Daxx, another permanent component of PML NBs, was reported to be involved in transcriptional regulation. Knocking down Daxx results in increased adenoviral and HCMV replication in cells (Tavalai et al., 2008; 
Schreiner et al., 2010).

PML deficiency renders mice more susceptible to some viral infection, including lymphocytic choriomeningitis virus, vesicular stomatitis virus (VSV) (Bonilla et al., 2002), encephalomyocarditis virus (El McHichi et al., 2010), resulting in an increased viral replication. Mouse embryonic fibroblasts (MEFs) derived from these mice are also more sensitive than wild type MEFs to rabies virus infection, while a high level of PML isoform IV leads to a reduction in rabies virus replication (Blondel et al., 2002). SUMOylation of PML IV is required for the antiviral effect (Blondel et al., 2010). Knocking down all PML isoforms by siRNA also significantly enhanced the propagation of influenza A virus strains PR8(H1N1), ST364(H3N2) (Li et al., 2009) and influenza virus in cells (Iki et al., 2005). Exogenous expression of PML III can also confer resistance to human foamy virus (HFV) (Rolley et al., 1995), VSV, influenza virus (Chelbi-Alix et al., 1998; Regad et al., 2001) and poliovirus infection (Pampin et al., 2006).

Although multiple PML isoforms are reported due to alternative splice of transcript, and most of isoforms localize in PML NBs, a subset of PML isoforms, lacking exons $5 \& 6$, named PML lb, exist in cytoplasm and is enriched during HSV-1 infection. PML Ib is demonstrated to sequestrate ICPO and mediate the intrinsic cellular defense against HSV-1 (McNally et al., 2008). Three of Sp100 isoforms could prevent the transcription of HSV-1 proteins ICPO and ICP4 at the promoter level, and IFN could change the splice of the Sp100 mRNA in favor of the inhibitor Sp100C (Negorev et al., 2009).

\section{Association of PML NBs with the viral genomes contributes to intrinsic resistance}

PML NBs have been implicated to inhibit the replication of adenovirus (Doucas et al., 1996) and limit early viral gene transcription of HSV-1 and HCMV shortly after virus entry (Everett et al., 2006; Tavalai et al., 2006; Everett et al., 2007). A number of studies show that PML NBs associate with the genome of several DNA viruses. Maul et al., for the first time, observed that the parental genomes of HSV-1, HCMV and adenovirus are associated with PML NBs (Ishov and Maul, 1996; Maul et al., 1996). Via examining newly-infected cells at the edges of developing virus plaques, PML NB component proteins were found to accumulate in PML NB like structures that are closely associated with the viral genomes, which only just enter the nucleus. PML and other PML NBs components were recruited to PML NB like structures during initial stages of infection, rather than the migration of pre-existing PML NBs (Wiesmeijer et al., 2002; Everett et al., 2004a; Everett and Murray, 2005). The process occurs extremely rapidly and does not depend on expression of viral protein, implying an intrinsic antiviral response to viral genome entry (Everett and Murray, 2005). The SUMO interaction motifs of PML, Sp100 and hDaxx are necessary for recruitment of these repressive proteins to HSV-1 genomes
(Cuchet-Lourenço et al., 2011).

The infection of mutant HSV-1 ICP0, ICP4 and/or VP16 causes quiescent infection which is stable and similar to latent infection. However, quiescent infection could be reversed only by provision of herpes viral proteins such as ICPO and not by alteration of cell physiological state. By establishing quiescent infection of mutant HSV-1 which inactivates ICPO, ICP4 and VP16, Everett et al. found that quiescent HSV-1 genomes in human fibroblast nucleus are associated with enlarged PML NB like structures, and the foci viral genomes were apparently enveloped within a sphere of PML and other ND10 proteins (Everett et al., 2007). During the initial stages of establishment of a quiescent infection in such cells, other ND10 proteins such as Sp100, hDaxx, and alpha thalassemia/mental retardation syndrome X-linked (ATRX) were recruited into PML NB like structures which associated with HSV-1 genomes. Accumulations of conjugated ubiquitin were also observed in PML NB like structures. Viral gene expression can be reactivated by superinfection with a virus that expresses ICPO. PML deficiency decreases the repression of ICPO-null mutant HSV-1(Everett and Chelbi-Alix, 2007) (Fig. 1).

Recently, PML NBs are reported to sequester newly assembled VZV nucleocapsids in neurons and satellite cells of human dorsal root ganglia and skin cells infected with VZV in vivo. PML fibers of those PML NBs form spherical cages that enclose mature and immature VZV nucleocapsids. Only PML IV could enhance the sequestration of nucleocapsids by interacting with VZV capsid surface protein, and significantly inhibit the viral infection by inhibiting nuclear egress and formation of infectious virus particles (Reichelt et al., 2011). Therefore PML cages were suggested to play a crucial role in the intrinsic antiviral defense, and the efficient sequestration of virion capsids in PML cages appears to be a basic cytoprotective function of PML NBs (Reichelt et al., 2011).

These observations illustrate a crucial role of PML NBs in the intrinsic antiviral defense and the potential link between PML NBs and quiescent infections. Recruitment of PML NBs components to sites associated with HSV-1 genomes and VZV nucleocapsids contributes to the intrinsic defense against invading viral genome (Everett et al., 2007; Cuchet-Lourenço et al., 2011; Reichelt et al., 2011), which suggests a crucial role of PML NBs in resistance to viral infection. However, the association of PML NBs with viral genome is complex in nature and requires further study. For example, Epstein-Barr virus genomes in latently infected cells do not appear to be associated with PML NBs (Bell et al., 2000).

Genomes of adenoviruses, simian virus 40 , papillomaviruses and polyomavirus were also shown to be associated with PML NBs. After entrance into the nucleus, parental genomes of those viruses move to the sites of pre-existing PML NBs, and the replication compartments of all these viruses become associated with PML NB like structures when viral 


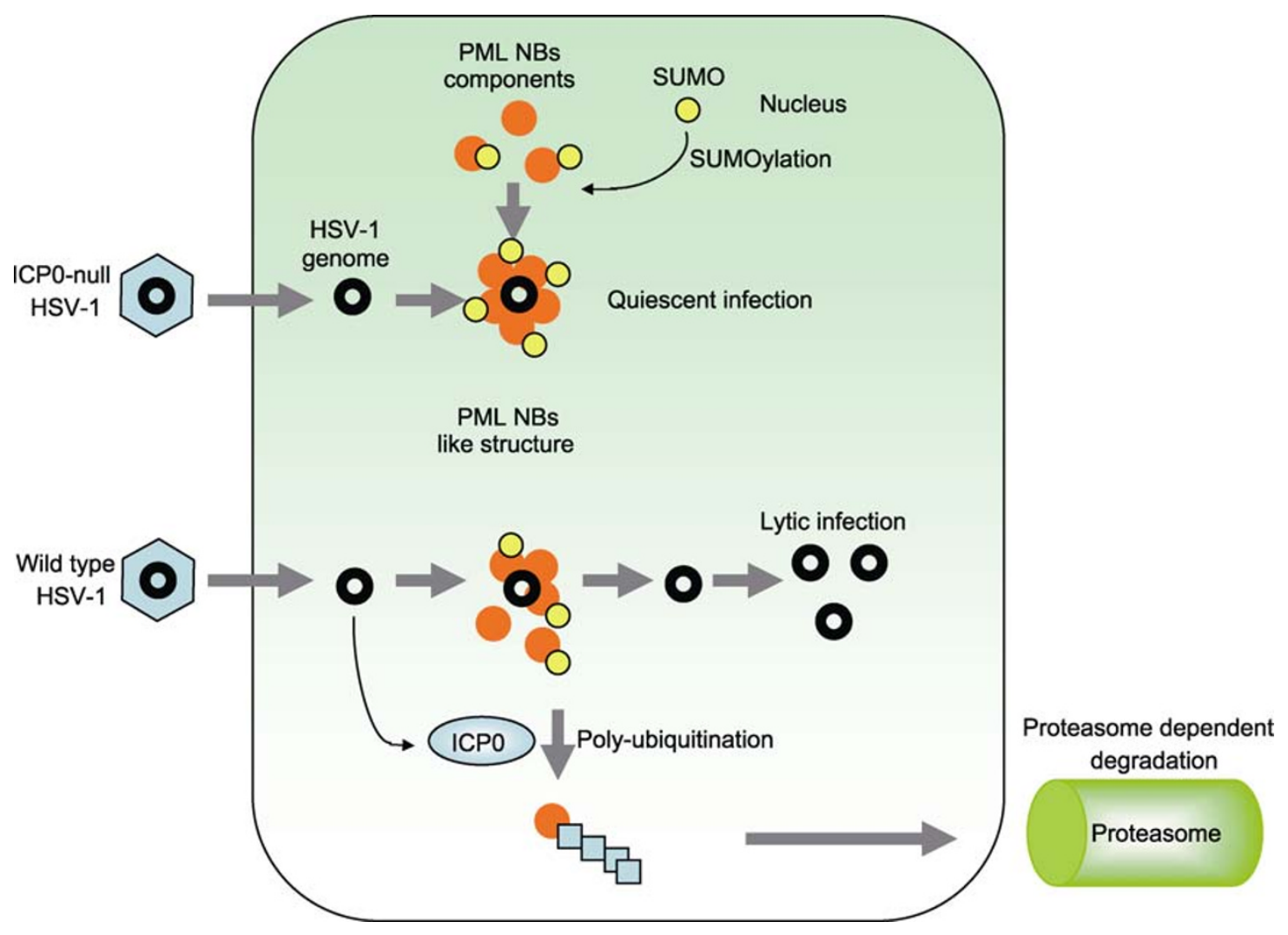

Figure 1. Association of PML NBs components with HSV-1 genomes contributes to intrinsic resistance. A sphere of PML NBs components in human fibroblast nucleus forms enlarged PML NBs like structures enveloping the HSV-1 genomes, and leading to the repression of HSV-1 genomes. The inhibition is counteracted by proteasome dependent degradation of PML NBs mediated by ICPO.

DNA replication begins (Doucas et al., 1996; Ishov et al., 1997; Maul, 1998; Everett, 2001; Fraefel et al., 2004; Jul-Larsen et al., 2004; Smith and Helenius, 2004).

\section{ICPO targets PML NBs}

The herpes viruses encode regulatory proteins that localize to and in many cases disrupt PML NB structure; the disruption of PML NBs by herpes virus regulatory proteins correlates with their functions in augmenting viral gene expression. Early studies have shown that ICPO localized in the nucleus at the onset of infection, with a punctate staining pattern in a diffuse background. It is also suggested that these ICPO foci corresponded to pre-existing cellular nuclear sub-structures PML NBs and that as infection progressed, ICPO had the amazing effect of completely disrupting them (Maul et al., 1993; Everett and Maul, 1994; Maul and Everett, 1994). Since PML staining disappears after HSV infection (Maul et al., 1993), it demonstrates that virus replication affects PML NBs.

The localization of ICPO to PML NBs is observed within a few hours post infection, followed by complete disruption of these structures, a process that requires both its RING finger domains conferring ICPO the ubiquitin E3 ligase activity. The
C-terminal region of ICPO is required for efficient localization of ICPO to PML NBs (Everett and Maul, 1994; Maul and Everett, 1994). The degradation of PML and the SUMOmodified isoforms of Sp100 is mediated by ICP0 in a proteasome-dependent manner (Boutell et al., 2002). Therefore, it is partly explained why exogenous expression of PML III or PML VI could not inhibit HSV-1 infection due to the proteasome dependent degradation of PML mediated by ICP0 (Chelbi-Alix and de Thé, 1999; Lopez et al., 2002). This process closely correlates with the role of ICPO in stimulating HSV-1 gene expression and lytic HSV-1 infection (Everett, 1988, 1989, 2000; Everett and Maul, 1994; Meredith et al., 1995; Hagglund and Roizman, 2004). It is reasonable to conclude that there is an intimate link between the ability of ICPO to interact with and disrupt PML NBs, and the regulation of latent and lytic infection by ICPO was in some way connected.

\section{PML/Sp100-related repression mechanism in the absence of ICPO}

To further investigate the relationship between ICPO and PML NBs, ICP0-null mutant viruses were constructed (Stow and Stow, 1986; Sacks and Schaffer, 1987). It was found then 
that in the absence of ICP0, the virus was still replication competitive but grew poorly and also reactivated from latency at much lower levels than the wild type virus when the multiplicity of infection (MOI) was low (Stow and Stow, 1986; Sacks and Schaffer 1987; Leib et al., 1989; Cai et al., 1993). Virus mutants lacking the ICPO gene have increased particle-to-PFU ratios, substantially lower yields, and decreased levels of gene expression and thus decreased probability of initiating lytic infection. ICP0-null mutant HSV-1 exhibits a greatly reduced plaque forming efficiency, but this defect is partially reversed in cells depleted of PML, Sp100, hDaxx or ATRX (Everett et al., 2006; Everett et al., 2008a).

Provision of exogenous ICPO allows reactivation of the quiescent genomes and entry into a normal productive cycle (Preston, 2000), strengthening the hypothesis that ICPO might be involved in the control of the balance between lytic and latent infection, such that in its absence the latent state is favored (Samaniego et al., 1998). The extent of this multiplicity dependent defect was cell-type dependent and can be overcome by cell cycle status (Cai and Schaffer, 1991; Yao and Schaffer, 1995), being particularly marked in limited-passage human fibroblasts, while less severe in BHK and Vero cells (Everett et al., 2004a) and negligible in osteosarcoma cell lines such as U2OS (Yao and Schaffer, 1995). Thus the defect could not be simply explained by damaged or faulty virus particles since apparently defective virus in one cell type was infectious in another (Yao and Schaffer, 1995). Furthermore, once lytic infection had been initiated at high $\mathrm{MOI}$, the mutant viruses produced normal levels of viral transcripts, proteins and progeny particles (Everett, 1989). However, using an equivalent number of viral particles could reduce the proportion of successfully infected cells by as much as 10,000 fold. It seemed that the ICPO mutant results in low probability of initiating lytic infection, and once the lytic infection had progressed beyond the early stages, it proceeded normally (Everett, 1989).

Cells have intrinsic defenses against virus infection, acting before the innate or the adaptive immune response. Pre-existing antiviral proteins such as PML, Sp100, and Daxx are stored in specific PML NBs. The antagonistic relationship between ICPO and components of PML NBs has implied the existence of certain link. During HSV-1 lytic infection, the viral regulatory protein ICPO localizes to PML NBs and induces the degradation of PML, thereby disrupting PML NBS and dispersing their constituent proteins whereas HSV mutants that fail to express ICPO are defective in their abilities to modify and degrade PML NBs components (Maul et al., 1993), leading to the consequence of latent infection.

The strong correlation between the effects of ICPO on PML NBs and its requirement for lytic virus infection prompted the hypothesis that PML NBs might have a repressive effect on HSV-1 gene expression and thereby constitute an intrinsic antiviral defense. ICP0-null mutant viruses are defective in PML degradation and PML NBs disruption, and concomi- tantly they initiate productive infection very inefficiently. The evidence that depletion of PML from human fibroblasts increases the probability of plaque formation and enhances gene expression of ICP0-null mutant HSV-1 (Everett et al., 2006) further supported the possibility that PML NBs structures have a repressive effect on viral infection, and viral regulatory protein ICPO that disrupts these structures does so to relieve this repression. Parallel studies using HCMV add weight to the hypothesis that PML and PML NBs contribute to an intrinsic cellular defense that represses herpes virus gene expression, which is countered by the activity of ICPO during Iytic infection (Everett, 2006; Everett and Chelbi-Alix, 2007). However, it is clear that PML cannot be the sole cellular factor involved in HSV-1 genome repression because the enhancement of ICPO-null mutant replication in PML-depleted cells is modest compared to that expected if repression were completely lifted (Everett et al., 2006).

Sp100, yet another major PML NBs component, has been implicated in repression of HSV-1 gene expression (Negorev et al., 2006) and in the regulation of Epstein-Barr virus transcription (Ling et al., 2005). It is confirmed that Sp100 is also involved in HSV-1 genome repression in the absence of ICPO in human fibroblasts by the observation that depletion of Sp100 results in a similar increase in ICP0-null mutant gene expression (Everett et al., 2008a).

Furthermore, depletion of PML or Sp100 from human fibroblasts modestly enhances ICPO-null mutant HSV-1 infection and gene expression. Deficiency of both proteins complements the mutant virus to a greater degree, but does not restore the plaque formation to wild type HSV-1 levels (Everett et al., 2006, 2008a). Those observations can be explained as both PML and Sp100 contribute to a cellular mechanism of HSV-1 genome repression; meanwhile, additional factors must be required for the remaining repression of ICP0-null mutant HSV-1 genomes that occurs in cells depleted of both PML and Sp100. Recently, Lukashchuk et al. revealed that knock down ATRX or Daxx by RNAi increases both gene expression and ICPO-null mutant HSV-1 and plaque formation. ATRX and Daxx act as a complex functioning in intrinsic antiviral resistance to HSV-1 infection. This process is also counteracted by ICPO (Lukashchuk and Everett, 2010).

Recently, E2FBP1/hDril1, an AT-rich interaction domain family protein, is reported to regulate the activation of $\mathrm{PML}$ NBs. E2FBP1 is capable of disintegrating PML NBs by specific deSumoylation of PML (Fukuyo et al., 2004). E2FBP1 could repress ICPO expression at the level of transcription, inhibiting accumulation of ICPO RNA; however, this process is counteracted by ICPO via polyubiquitylation of E2FBP1 mediated by RING/HUL-2 (herpes ubiquitin ligase 2) domain of ICP0. E2FBP1 interacts with ICPO in vivo, and affects ICPO's nuclear distribution (Fukuyo et al., 2011). Those observations suggest that E2FBP1 functions as an intrinsic cellular defense factor in spite of its PML NB dissociation 
function, also outmaneuvered by ICPO.

A logical explanation for this phenomenon that HSV-1 originates the lytic infection from latent state in virtue of ICPO may lie in resisting the host suicide or self-protection system, because cell death is detrimental for viral proliferation. On the one hand, ICPO inhibits the breakage of PML NBs structure by E2FBP1 to repress the premature senescence; on the other hand, ICPO could mediate the dissociation of PML NBs itself in HSV-1 infected cells. The direct or indirect relationship between ICPO and PML NBs may be out of HSV-1 life-cycle demand. The key point for ICP0 to regulate the PML NBs function is exactly mediated during various HSV-1 life periods.

The orthologues of ICPO expressed by other alphaherpes viruses have similar biological functions and can disrupt PML NBs, including BICPO of bovine herpes virus 1, Eg63 of equine herpes virus 1, ORF61 protein in VZV and EP0 in pseudorabies virus (PRV) (Parkinson and Everett, 2000).

In a word, all the previous studies and observations establish the following inferences: (1) Components of PML NBs contribute to intrinsic anti-HSV defense mechanism; (2) E3 ubiquitin ligase activity of ICPO counterbalances the cellular repression by proteasomal degradation of PML NBs, promoting viral gene expression and switching into lytic cycle; (3) Cells infected with ICP0-null mutant viruses fail to degrade repressive factors, resulting in quiescent status of viral genomes, which is the characteristic of latency; (4) The evidence that depletion of PML enhances gene expression of ICPO-null mutant HSV-1 demonstrates that the link between PML NBs and ICPO in regulating lytic and latent infection of HSV-1 does exactly exist.

\section{CONCLUSIONS}

The observations that HSV-1 mutants, failing to express the viral immediate-early protein ICPO, have a pronounced defect in viral gene expression and efficient progression of infected cells into lytic infection, especially at low $\mathrm{MOI}$, have led to the inference that ICPO produces a marked effect on the regulation of the balance between lytic and latent HSV-1 infection.

ICP0, acting as a wide-spectrum transactivator of gene expression and the ubiquitin E3 ligase activity conferred by the RING finger domain, is one of the most prominent activities, with the ability to localize to and disrupt discrete nuclear structures known as PML NBs or ND10. This disruption occurs through ICP0-induced degradation of PML. HSV-1 mutants that fail to express ICPO or that express mutant ICPO proteins that lack RING finger activity are unable to disrupt PML NBs or to degrade PML. Such mutants have a profound defect in HSV-1 gene expression after infection of limited-passage human fibroblasts. With the data above, a hypothesis that degradation of PML NBs plays a key role in progressing infected cells into productive infection arose, to which the accumulating evidence that several PML NBs pro- teins, including PML and Sp100, are involved in the repression or regulation of viral gene expression lends support. All together, we conclude that there is a potential link between PML and Sp100—contributing to repression of HSV-1 gene expression and the functions of ICPO in regulating lytic and latent infection. Understanding the mechanisms by which ICPO represses intrinsic resistance will provide new insight into the pivotal role of ICP0 during HSV-1 infection, making ICPO an attractive target for designing antiviral drug to prevent HSV-1 diseases.

\section{ACKNOWLEDGEMENTS}

This study was supported by grants from the State Key Development Program for Basic Research of China (973 Program) (Grant Nos. 2010CB530105 and 2011CB504802); The Startup Fund of the 100 Talents Program from the Chinese Academy of Sciences (No. 20072020-141); National Natural Science Foundation of China (Grant Nos. 30870120, 81171584, 81101263, and 81000736)

We apologize to those in the field whose work was not cited here owing to space limitations. We are grateful to anonymous reviewers for their help to improve this review.

\section{ABBREVIATIONS}

BLM, the Bloom syndrome helicase; Daxx, the death domain-associated protein; HCMV, human cytomegalovirus; HFV, human foamy virus; HSV-1, herpes simplex virus type 1; IE, immediate-early; LATs, latency-associated transcripts; MEFs, mouse embryonic fibroblasts; MOI, multiplicity of infection; PML NBs, promyelocytic leukemia (PML) nuclear bodies (NBs); PRV, pseudorabies virus; SUMO, small ubiquitin-like modifier; VSV, vesicular stomatitis virus; VZV, varicella-zoster virus

\section{REFERENCES}

Ahn, J.H., and Hayward, G.S. (2000). Disruption of PML-associated nuclear bodies by IE1 correlates with efficient early stages of viral gene expression and DNA replication in human cytomegalovirus infection. Virology 274, 39-55.

Antón, L.C., Schubert, U., Bacík, I., Princiotta, M.F., Wearsch, P.A., Gibbs, J., Day, P.M., Realini, C., Rechsteiner, M.C., Bennink, J.R., et al. (1999). Intracellular localization of proteasomal degradation of a viral antigen. J Cell Biol 146, 113-124.

Aslani, A., Simonsson, S., and Elias, P. (2000). A novel conformation of the herpes simplex virus origin of DNA replication recognized by the origin binding protein. J Biol Chem 275, 5880-5887.

Bell, P., Lieberman, P.M., and Maul, G.G. (2000). Lytic but not latent replication of epstein-barr virus is associated with PML and induces sequential release of nuclear domain 10 proteins. J Virol 74 , 11800-11810.

Bernardi, R., and Pandolfi, P.P. (2003). Role of PML and the PML-nuclear body in the control of programmed cell death. Oncogene 22, 9048-9057. 
Bischof, O., Kirsh, O., Pearson, M., Itahana, K., Pelicci, P.G., and Dejean, A. (2002). Deconstructing PML-induced premature senescence. EMBO J 21, 3358-3369.

Blondel, D., Kheddache, S., Lahaye, X., Dianoux, L., and Chelbi-Alix, M.K. (2010). Resistance to rabies virus infection conferred by the PMLIV isoform. J Virol 84, 10719-10726.

Blondel, D., Regad, T., Poisson, N., Pavie, B., Harper, F., Pandolfi, P.P., De Thé, H., and Chelbi-Alix, M.K. (2002). Rabies virus $P$ and small $\mathrm{P}$ products interact directly with $\mathrm{PML}$ and reorganize $\mathrm{PML}$ nuclear bodies. Oncogene 21, 7957-7970.

Bonilla, W.V., Pinschewer, D.D., Klenerman, P., Rousson, V., Gaboli, M., Pandolfi, P.P., Zinkernagel, R.M., Salvato, M.S., and Hengartner, H. (2002). Effects of promyelocytic leukemia protein on virus-host balance. J Virol 76, 3810-3818.

Boutell, C., and Everett, R.D. (2003). The herpes simplex virus type 1 (HSV-1) regulatory protein ICPO interacts with and Ubiquitinates p53. J Biol Chem 278, 36596-36602.

Boutell, C., Sadis, S., and Everett, R.D. (2002). Herpes simplex virus type 1 immediate-early protein ICPO and is isolated RING finger domain act as ubiquitin E3 ligases in vitro. J Virol 76, 841-850.

Cai, W., Astor, T.L., Liptak, L.M., Cho, C., Coen, D.M., and Schaffer, P.A. (1993). The herpes simplex virus type 1 regulatory protein ICPO enhances virus replication during acute infection and reactivation from latency. J Virol 67, 7501-7512.

Cai, W., and Schaffer, P.A. (1991). A cellular function can enhance gene expression and plating efficiency of a mutant defective in the gene for ICPO, a transactivating protein of herpes simplex virus type 1. J Virol 65, 4078-4090.

Cai, W., and Schaffer, P.A. (1992). Herpes simplex virus type 1 ICPO regulates expression of immediate-early, early, and late genes in productively infected cells. J Virol 66, 2904-2915.

Chelbi-Alix, M.K., and de Thé, H. (1999). Herpes virus induced proteasome-dependent degradation of the nuclear bodies-associated PML and Sp100 proteins. Oncogene 18, 935-941.

Chelbi-Alix, M.K., Quignon, F., Pelicano, L., Koken, M.H., and de Thé, H. (1998). Resistance to virus infection conferred by the interferon-induced promyelocytic leukemia protein. J Virol 72, 1043-1051.

Cuchet-Lourenço, D., Boutell, C., Lukashchuk, V., Grant, K., Sykes, A., Murray, J., Orr, A., and Everett, R.D. (2011). SUMO pathway dependent recruitment of cellular repressors to herpes simplex virus type 1 genomes. PLoS Pathog 7, e1002123.

Dellaire, G., and Bazett-Jones, D.P. (2004). PML nuclear bodies: dynamic sensors of DNA damage and cellular stress. Bioessays 26, 963-977.

Dellaire, G., Ching, R.W., Ahmed, K., Jalali, F., Tse, K.C., Bristow, R.G., and Bazett-Jones, D.P. (2006). Promyelocytic leukemia nuclear bodies behave as DNA damage sensors whose response to DNA double-strand breaks is regulated by NBS1 and the kinases ATM, Chk2, and ATR. J Cell Biol 175, 55-66.

DeLuca, N.A., McCarthy, A.M., and Schaffer, P.A. (1985). Isolation and characterization of deletion mutants of herpes simplex virus type 1 in the gene encoding immediate-early regulatory protein ICP4. J Virol 56, 558-570.

Doucas, V., Ishov, A.M., Romo, A., Juguilon, H., Weitzman, M.D., Evans, R.M., and Maul, G.G. (1996). Adenovirus replication is coupled with the dynamic properties of the PML nuclear structure. Genes Dev 10, 196-207.

Duprez, E., Saurin, A.J., Desterro, J.M., Lallemand-Breitenbach, V., Howe, K., Boddy, M.N., Solomon, E., de Thé, H., Hay, R.T., and Freemont, P.S. (1999). SUMO-1 modification of the acute promyelocytic leukaemia protein PML: implications for nuclear localisation. J Cell Sci 112, 381-393.

Efstathiou, S., and Preston, C.M. (2005). Towards an understanding of the molecular basis of herpes simplex virus latency. Virus Res 111, 108-119.

El McHichi, B., Regad, T., Maroui, M.A., Rodriguez, M.S., Aminev, A., Gerbaud, S., Escriou, N., Dianoux, L., and Chelbi-Alix, M.K. (2010). SUMOylation promotes PML degradation during encephalomyocarditis virus infection. J Virol 84, 11634-11645.

Everett, R.D. (1988). Analysis of the functional domains of herpes simplex virus type 1 immediate-early polypeptide Vmw110. J Mol Biol 202, 87-96.

Everett, R.D. (1989). Construction and characterization of herpes simplex virus type 1 mutants with defined lesions in immediate early gene 1. J Gen Virol 70, 1185-1202.

Everett, R.D. (2000). ICP0, a regulator of herpes simplex virus during lytic and latent infection. Bioessays 22, 761-770.

Everett, R.D. (2001). DNA viruses and viral proteins that interact with PML nuclear bodies. Oncogene 20, 7266-7273.

Everett, R.D. (2006). Interactions between DNA viruses, ND10 and the DNA damage response. Cell Microbiol 8, 365-374.

Everett, R.D., Boutell, C., and Orr, A. (2004a). Phenotype of a herpes simplex virus type 1 mutant that fails to express immediate-early regulatory protein ICP0. J Virol 78, 1763-1774.

Everett, R.D., and Chelbi-Alix, M.K. (2007). PML and PML nuclear bodies: implications in antiviral defence. Biochimie 89, 819-830.

Everett, R.D., Earnshaw, W.C., Findlay, J., and Lomonte, P. (1999a). Specific destruction of kinetochore protein CENP-C and disruption of cell division by herpes simplex virus immediate-early protein Vmw110. EMBO J 18, 1526-1538.

Everett, R.D., Freemont, P., Saitoh, H., Dasso, M., Orr, A., Kathoria, M., and Parkinson, J. (1998a). The disruption of ND10 during herpes simplex virus infection correlates with the Vmw110- and proteasome-dependent loss of several PML isoforms. J Virol 72, $6581-6591$.

Everett, R.D., Lomonte, P., Sternsdorf, T., van Driel, R., and Orr, A. (1999b). Cell cycle regulation of PML modification and ND10 composition. J Cell Sci 112, 4581-4588.

Everett, R.D., and Maul, G.G. (1994). HSV-1 IE protein Vmw110 causes redistribution of PML. EMBO J 13, 5062-5069.

Everett, R.D., Meredith, M., Orr, A., Cross, A., Kathoria, M., and Parkinson, J. (1997). A novel ubiquitin-specific protease is dynamically associated with the PML nuclear domain and binds to a herpesvirus regulatory protein. EMBO J 16, 566-577.

Everett, R.D., and Murray, J. (2005). ND10 components relocate to sites associated with herpes simplex virus type 1 nucleoprotein complexes during virus infection. J Virol 79, 5078-5089.

Everett, R.D., Murray, J., Orr, A., and Preston, C.M. (2007). Herpes simplex virus type 1 genomes are associated with ND10 nuclear substructures in quiescently infected human fibroblasts. J Virol 81 , 10991-11004. 
Everett, R.D., Orr, A., and Preston, C.M. (1998b). A viral activator of gene expression functions via the ubiquitin-proteasome pathway. EMBO J 17, 7161-7169.

Everett, R.D., Parada, C., Gripon, P., Sirma, H., and Orr, A. (2008a). Replication of ICP0-null mutant herpes simplex virus type 1 is restricted by both PML and Sp100. J Virol 82, 2661-2672.

Everett, R.D., Rechter, S., Papior, P., Tavalai, N., Stamminger, T., and Orr, A. (2006). PML contributes to a cellular mechanism of repression of herpes simplex virus type 1 infection that is inactivated by ICP0. J Virol 80, 7995-8005.

Everett, R.D., Sourvinos, G., Leiper, C., Clements, J.B., and Orr, A. (2004b). Formation of nuclear foci of the herpes simplex virus type 1 regulatory protein ICP4 at early times of infection: localization, dynamics, recruitment of ICP27, and evidence for the de novo induction of ND10-like complexes. J Virol 78, 1903-1917.

Everett, R.D., Young, D.F., Randall, R.E., and Orr, A. (2008b). STAT-1- and IRF-3-dependent pathways are not essential for repression of ICP0-null mutant herpes simplex virus type 1 in human fibroblasts. J Virol 82, 8871-8881.

Ferenczy, M.W., and DeLuca, N.A. (2011). Reversal of heterochromatic silencing of quiescent herpes simplex virus type 1 by ICP0. J Virol 85, 3424-3435.

Fraefel, C., Bittermann, A.G., Büeler, H., Heid, I., Bächi, T., and Ackermann, M. (2004). Spatial and temporal organization of adeno-associated virus DNA replication in live cells. J Virol 78, 389-398.

Fukuyo, Y., Horikoshi, N., Ishov, A.M., Silverstein, S.J., and Nakajima, T. (2011). The herpes simplex virus immediate-early ubiquitin ligase ICPO induces degradation of the ICPO repressor protein E2FBP1. J Virol 85, 3356-3366.

Fukuyo, Y., Mogi, K., Tsunematsu, Y., and Nakajima, T. (2004). E2FBP1/hDril1 modulates cell growth through downregulation of promyelocytic leukemia bodies. Cell Death Differ 11, 747-759.

Gresko, E., Ritterhoff, S., Sevilla-Perez, J., Roscic, A., Fröbius, K., Kotevic, I., Vichalkovski, A., Hess, D., Hemmings, B.A., and Schmitz, M.L. (2009). PML tumor suppressor is regulated by HIPK2-mediated phosphorylation in response to DNA damage. Oncogene 28, 698-708.

$\mathrm{Gu}, \mathrm{H}$., and Roizman, B. (2003). The degradation of promyelocytic leukemia and Sp100 proteins by herpes simplex virus 1 is mediated by the ubiquitin-conjugating enzyme $\mathrm{UbcH} 5 \mathrm{a}$. Proc Natl Acad Sci U S A 100, 8963-8968.

Hagglund, R., and Roizman, B. (2002). Characterization of the novel E3 ubiquitin ligase encoded in exon 3 of herpes simplex virus-1-infected cell protein 0 . Proc Natl Acad Sci U S A 99, 7889-7894.

Hagglund, R., and Roizman, B. (2003). Herpes simplex virus 1 mutant in which the ICPO HUL-1 E3 ubiquitin ligase site is disrupted stabilizes cdc34 but degrades D-type cyclins and exhibits diminished neurotoxicity. J Virol 77, 13194-13202.

Hagglund, R., and Roizman, B. (2004). Role of ICPO in the strategy of conquest of the host cell by herpes simplex virus 1. J Virol 78, 2169-2178.

Hagglund, R., Van Sant, C., Lopez, P., and Roizman, B. (2002). Herpes simplex virus 1 -infected cell protein 0 contains two E3 ubiquitin ligase sites specific for different E2 ubiquitin-conjugating enzymes. Proc Natl Acad Sci U S A 99, 631-636.

Halford, W.P., Kemp, C.D., Isler, J.A., Davido, D.J., and Schaffer, P.A (2001). ICP0, ICP4, or VP16 expressed from adenovirus vectors induces reactivation of latent herpes simplex virus type 1 in primary cultures of latently infected trigeminal ganglion cells. J Virol $75,6143-6153$.

Halford, W.P., and Schaffer, P.A. (2001). ICP0 is required for efficient reactivation of herpes simplex virus type 1 from neuronal latency. J Virol 75, 3240-3249.

Harris, R.A., and Preston, C.M. (1991). Establishment of latency in vitro by the herpes simplex virus type 1 mutant in1814. J Gen Virol 72, 907-913.

Herrera, F.J., and Triezenberg, S.J. (2004). VP16-dependent association of chromatin-modifying coactivators and underrepresentation of histones at immediate-early gene promoters during herpes simplex virus infection. J Virol 78, 9689-9696.

Iki, S., Yokota, S., Okabayashi, T., Yokosawa, N., Nagata, K., and Fujii, N. (2005). Serum-dependent expression of promyelocytic leukemia protein suppresses propagation of influenza virus. Virology 343, 106-115.

Ishov, A.M., and Maul, G.G. (1996). The periphery of nuclear domain 10 (ND10) as site of DNA virus deposition. J Cell Biol 134, 815-826.

Ishov, A.M., Sotnikov, A.G., Negorev, D., Vladimirova, O.V., Neff, N., Kamitani, T., Yeh, E.T., Strauss, J.F. 3rd, and Maul, G.G. (1999). PML is critical for ND10 formation and recruits the PML-interacting protein daxx to this nuclear structure when modified by SUMO-1. J Cell Biol 147, 221-234.

Ishov, A.M., Stenberg, R.M., and Maul, G.G. (1997). Human cytomegalovirus immediate early interaction with host nuclear structures: definition of an immediate transcript environment. J Cell Biol 138, 5-16.

Jackson, S.A., and DeLuca, N.A. (2003). Relationship of herpes simplex virus genome configuration to productive and persistent infections. Proc Natl Acad Sci U S A 100, 7871-7876.

Jul-Larsen, A., Visted, T., Karlsen, B.O., Rinaldo, C.H., Bjerkvig, R., Lønning, P.E., and Bøe, S.O. (2004). PML-nuclear bodies accumulate DNA in response to polyomavirus $\mathrm{BK}$ and simian virus 40 replication. Exp Cell Res 298, 58-73.

Kamitani, T., Kito, K., Nguyen, H.P., Wada, H., Fukuda-Kamitani, T., and Yeh, E.T. (1998a). Identification of three major sentrinization sites in PML. J Biol Chem 273, 26675-26682.

Kamitani, T., Nguyen, H.P., Kito, K., Fukuda-Kamitani, T., and Yeh, E.T. (1998b). Covalent modification of PML by the sentrin family of ubiquitin-like proteins. J Biol Chem 273, 3117-3120.

Kawaguchi, Y., Bruni, R., and Roizman, B. (1997a). Interaction of herpes simplex virus 1 alpha regulatory protein ICP0 with elongation factor 1delta: ICP0 affects translational machinery. J Virol 71, 1019-1024.

Kawaguchi, Y., Tanaka, M., Yokoymama, A., Matsuda, G., Kato, K., Kagawa, H., Hirai, K., and Roizman, B. (2001). Herpes simplex virus 1 alpha regulatory protein ICPO functionally interacts with cellular transcription factor BMAL1. Proc Natl Acad Sci U S A 98, 1877-1882.

Kawaguchi, Y., Van Sant, C., and Roizman, B. (1997b). Herpes simplex virus 1 alpha regulatory protein ICPO interacts with and 
stabilizes the cell cycle regulator cyclin D3. J Virol 71, 7328-7336.

Klemm, R.D., Goodrich, J.A., Zhou, S., and Tjian, R. (1995). Molecular cloning and expression of the 32-kDa subunit of human TFIID reveals interactions with VP16 and TFIIB that mediate transcriptional activation. Proc Natl Acad Sci U S A 92, 5788-5792.

Kyratsous, C.A., and Silverstein, S.J. (2009). Components of nuclear domain 10 bodies regulate varicella-zoster virus replication. J Virol 83, 4262-4274.

Lallemand-Breitenbach, V., Zhu, J., Puvion, F., Koken, M., Honoré, N., Doubeikovsky, A., Duprez, E., Pandolfi, P.P., Puvion, E., Freemont, P., et al. (2001). Role of promyelocytic leukemia (PML) sumolation in nuclear body formation, $11 \mathrm{~S}$ proteasome recruitment, and As2O3-induced PML or PML/retinoic acid receptor alpha degradation. J Exp Med 193, 1361-1371.

Lees-Miller, S.P., Long, M.C., Kilvert, M.A., Lam, V., Rice, S.A., and Spencer, C.A. (1996). Attenuation of DNA-dependent protein kinase activity and its catalytic subunit by the herpes simplex virus type 1 transactivator ICP0. J Virol 70, 7471-7477.

Lehman, I.R., and Boehmer, P.E. (1999). Replication of herpes simplex virus DNA. J Biol Chem 274, 28059-28062.

Leib, D.A., Coen, D.M., Bogard, C.L., Hicks, K.A., Yager, D.R., Knipe, D.M., Tyler, K.L., and Schaffer, P.A. (1989). Immediate-early regulatory gene mutants define different stages in the establishment and reactivation of herpes simplex virus latency. J Virol 63, 759-768.

Li, W., Wang, G., Zhang, H., Zhang, D., Zeng, J., Chen, X., Xu, Y., and $\mathrm{Li}, \mathrm{K}$. (2009). Differential suppressive effect of promyelocytic leukemia protein on the replication of different subtypes/strains of influenza A virus. Biochem Biophys Res Commun 389, 84-89.

Ling, P.D., Peng, R.S., Nakajima, A., Yu, J.H., Tan, J., Moses, S.M., Yang, W.H., Zhao, B., Kieff, E., Bloch, K.D., et al. (2005). Mediation of Epstein-Barr virus EBNA-LP transcriptional coactivation by Sp100. EMBO J 24, 3565-3575.

Lomonte, P., and Morency, E. (2007). Centromeric protein CENP-B proteasomal degradation induced by the viral protein ICPO. FEBS Lett 581, 658-662.

Lomonte, P., Sullivan, K.F., and Everett, R.D. (2001). Degradation of nucleosome-associated centromeric histone $\mathrm{H} 3$-like protein CENP-A induced by herpes simplex virus type 1 protein ICPO. J Biol Chem 276, 5829-5835.

Lomonte, P., Thomas, J., Texier, P., Caron, C., Khochbin, S., and Epstein, A.L. (2004). Functional interaction between class II histone deacetylases and ICP0 of herpes simplex virus type 1. J Virol 78, 6744-6757.

Lopez, P., Jacob, R.J., and Roizman, B. (2002). Overexpression of promyelocytic leukemia protein precludes the dispersal of ND10 structures and has no effect on accumulation of infectious herpes simplex virus 1 or its proteins. J Virol 76, 9355-9367.

Lukashchuk, V., and Everett, R.D. (2010). Regulation of ICP0-null mutant herpes simplex virus type 1 infection by ND10 components ATRX and hDaxx. J Virol 84, 4026-4040.

Mahajan, S.S., Little, M.M., Vazquez, R., and Wilson, A.C. (2002). Interaction of HCF-1 with a cellular nuclear export factor. J Biol Chem 277, 44292-44299.

Maul, G.G. (1998). Nuclear domain 10, the site of DNA virus tran- scription and replication. Bioessays 20,660-667.

Maul, G.G., and Everett, R.D. (1994). The nuclear location of PML, a cellular member of the $\mathrm{C} 3 \mathrm{HC} 4$ zinc-binding domain protein family, is rearranged during herpes simplex virus infection by the $\mathrm{C} 3 \mathrm{HC} 4$ viral protein ICP0. J Gen Virol 75, 1223-1233.

Maul, G.G., Guldner, H.H., and Spivack, J.G. (1993). Modification of discrete nuclear domains induced by herpes simplex virus type 1 immediate early gene 1 product (ICP0). J Gen Virol 74, 2679-2690.

Maul, G.G., Ishov, A.M., and Everett, R.D. (1996). Nuclear domain 10 as preexisting potential replication start sites of herpes simplex virus type-1. Virology 217, 67-75.

McNally, B.A., Trgovcich, J., Maul, G.G., Liu, Y., and Zheng, P. (2008). A role for cytoplasmic PML in cellular resistance to viral infection. PLoS One 3, e2277.

Memedula, S., and Belmont, A.S. (2003). Sequential recruitment of HAT and SWI/SNF components to condensed chromatin by VP16. Curr Biol 13, 241-246.

Meredith, M., Orr, A., Elliott, M., and Everett, R. (1995). Separation of sequence requirements for HSV-1 Vmw110 multimerisation and interaction with a 135-kDa cellular protein. Virology 209, 174-187.

Mitchell, B.M., Bloom, D.C., Cohrs, R.J., Gilden, D.H., and Kennedy, P.G. (2003). Herpes simplex virus-1 and varicella-zoster virus latency in ganglia. J Neurovirol 9, 194-204.

Mittler, G., Stühler, T., Santolin, L., Uhlmann, T., Kremmer, E., Lottspeich, F., Berti, L., and Meisterernst, M. (2003). A novel docking site on Mediator is critical for activation by VP16 in mammalian cells. EMBO J 22, 6494-6504.

Mossman, K.L., and Smiley, J.R. (2002). Herpes simplex virus ICP0 and ICP34.5 counteract distinct interferon-induced barriers to virus replication. J Virol 76, 1995-1998.

Müller, S., Matunis, M.J., and Dejean, A. (1998). Conjugation with the ubiquitin-related modifier SUMO-1 regulates the partitioning of PML within the nucleus. EMBO J 17, 61-70.

Negorev, D., and Maul, G.G. (2001). Cellular proteins localized at and interacting within ND10/PML nuclear bodies/PODs suggest functions of a nuclear depot. Oncogene 20, 7234-7242.

Negorev, D.G., Vladimirova, O.V., Ivanov, A., Rauscher, F. 3rd, and Maul, G.G. (2006). Differential role of Sp100 isoforms in interferon-mediated repression of herpes simplex virus type 1 immediate-early protein expression. J Virol 80, 8019-8029.

Negorev, D.G., Vladimirova, O.V., and Maul, G.G. (2009). Differential functions of interferon-upregulated Sp100 isoforms: herpes simplex virus type 1 promoter-based immediate-early gene suppression and PML protection from ICP0-mediated degradation. J Virol 83, 5168-5180.

Pampin, M., Simonin, Y., Blondel, B., Percherancier, Y., and Chelbi-Alix, M.K. (2006). Cross talk between PML and p53 during poliovirus infection: implications for antiviral defense. J Virol 80, 8582-8592.

Parkinson, J., and Everett, R.D. (2000). Alphaherpesvirus proteins related to herpes simplex virus type 1 ICPO affect cellular structures and proteins. J Virol 74, 10006-10017.

Parkinson, J., Lees-Miller, S.P., and Everett, R.D. (1999). Herpes simplex virus type 1 immediate-early protein vmw110 induces the proteasome-dependent degradation of the catalytic subunit of 
DNA-dependent protein kinase. J Virol 73, 650-657.

Paterson, T., and Everett, R.D. (1988). Mutational dissection of the HSV-1 immediate-early protein Vmw175 involved in transcriptional transactivation and repression. Virology 166, 186-196.

Preston, C.M. (2000). Repression of viral transcription during herpes simplex virus latency. J Gen Virol 81, 1-19.

Preston, C.M., and Nicholl, M.J. (1997). Repression of gene expression upon infection of cells with herpes simplex virus type $1 \mathrm{mu}-$ tants impaired for immediate-early protein synthesis. J Virol 71, 7807-7813.

Regad, T., and Chelbi-Alix, M.K. (2001). Role and fate of PML nuclear bodies in response to interferon and viral infections. Oncogene 20, 7274-7286.

Regad, T., Saib, A., Lallemand-Breitenbach, V., Pandolfi, P.P., de Thé, H., and Chelbi-Alix, M.K. (2001). PML mediates the interferon-induced antiviral state against a complex retrovirus via its association with the viral transactivator. EMBO J 20, 3495-3505.

Reichelt, M., Wang, L., Sommer, M., Perrino, J., Nour, A.M., Sen, N., Baiker, A., Zerboni, L., and Arvin, A.M. (2011). Entrapment of viral capsids in nuclear PML cages is an intrinsic antiviral host defense against varicella-zoster virus. PLoS Pathog 7, e1001266.

Rolley, N., Butcher, S., and Milner, J. (1995). Specific DNA binding by different classes of human p53 mutants. Oncogene 11, 763-770.

Sacks, W.R., and Schaffer, P.A. (1987). Deletion mutants in the gene encoding the herpes simplex virus type 1 immediate-early protein ICP0 exhibit impaired growth in cell culture. J Virol 61, 829-839.

Saffert, R.T., and Kalejta, R.F. (2008). Promyelocytic leukemia-nuclear body proteins: herpesvirus enemies, accomplices, or both? Future Virol 3, 265-277.

Samaniego, L.A., Neiderhiser, L., and DeLuca, N.A. (1998). Persistence and expression of the herpes simplex virus genome in the absence of immediate-early proteins. J Virol 72, 3307-3320.

Schreiner, S., Wimmer, P., Sirma, H., Everett, R.D., Blanchette, P., Groitl, P., and Dobner, T. (2010). Proteasome-dependent degradation of Daxx by the viral E1B-55K protein in human adenovirus-infected cells. J Virol 84, 7029-7038.

Seeler, J.S., and Dejean, A. (2001). SUMO: of branched proteins and nuclear bodies. Oncogene 20, 7243-7249.

Shen, T.H., Lin, H.K., Scaglioni, P.P., Yung, T.M., and Pandolfi, P.P. (2006). The mechanisms of PML-nuclear body formation. Mol Cell 24, 331-339.

Shimomura Y. (2008). Herpes simplex virus latency, reactivation, and a new antiviral therapy for herpetic keratitis. Nihon Ganka Gakkai Zasshi 112, 247-264; discussion 265.

Smith, A.E., and Helenius, A. (2004). How viruses enter animal cells. Science 304, 237-242.

Smith, C.A., Bates, P., Rivera-Gonzalez, R., Gu, B., and DeLuca, N.A. (1993). ICP4, the major transcriptional regulatory protein of herpes simplex virus type 1 , forms a tripartite complex with TATA-binding protein and TFIIB. J Virol 67, 4676-4687.

Sternsdorf, T., Grötzinger, T., Jensen, K., and Will, H. (1997a). Nuclear dots: actors on many stages. Immunobiology 198, 307-331.

Sternsdorf, T., Jensen, K., Reich, B., and Will, H. (1999). The nuclear dot protein sp100, characterization of domains necessary for dimerization, subcellular localization, and modification by small ubiquitin-like modifiers. J Biol Chem 274, 12555-12566.

Sternsdorf, T., Jensen, K., and Will, H. (1997b). Evidence for covalent modification of the nuclear dot-associated proteins PML and Sp100 by PIC1/SUMO-1. J Cell Biol 139, 1621-1634.

Stow, N.D., and Stow, E.C. (1986). Isolation and characterization of a herpes simplex virus type 1 mutant containing a deletion within the gene encoding the immediate early polypeptide Vmw110. J Gen Virol 67, 2571-2585.

Tavalai, N., Papior, P., Rechter, S., Leis, M., and Stamminger, T. (2006). Evidence for a role of the cellular ND10 protein PML in mediating intrinsic immunity against human cytomegalovirus infections. J Virol 80, 8006-8018.

Tavalai, N., Papior, P., Rechter, S., and Stamminger, T. (2008). Nuclear domain 10 components promyelocytic leukemia protein and hDaxx independently contribute to an intrinsic antiviral defense against human cytomegalovirus infection. J Virol 82, 126-137.

Tavalai, N., and Stamminger, T. (2008). New insights into the role of the subnuclear structure ND10 for viral infection. Biochim Biophys Acta 1783, 2207-2221.

Van Sant, C., Hagglund, R., Lopez, P., and Roizman, B. (2001). The infected cell protein 0 of herpes simplex virus 1 dynamically interacts with proteasomes, binds and activates the cdc34 E2 ubiquitin-conjugating enzyme, and possesses in vitro E3 ubiquitin ligase activity. Proc Natl Acad Sci U S A 98, 8815-8820.

von Einem, J., Schumacher, D., O'Callaghan, D.J., and Osterrieder, N. (2006). The alpha-TIF (VP16) homologue (ETIF) of equine herpesvirus 1 is essential for secondary envelopment and virus egress. J Virol 80, 2609-2620.

Wagner, E.K., and Bloom, D.C. (1997). Experimental investigation of herpes simplex virus latency. Clin Microbiol Rev 10, 419-443.

Watson, R.J., and Clements, J.B. (1980). A herpes simplex virus type 1 function continuously required for early and late virus RNA synthesis. Nature 285, 329-330.

Wiesmeijer, K., Molenaar, C., Bekeer, I.M., Tanke, H.J., and Dirks, R.W. (2002). Mobile foci of Sp100 do not contain PML: PML bodies are immobile but PML and Sp100 proteins are not. J Struct Biol 140, 180-188.

Wysocka, J., and Herr, W. (2003). The herpes simplex virus VP16-induced complex: the makings of a regulatory switch. Trends Biochem Sci 28, 294-304.

Xiao, H., Pearson, A., Coulombe, B., Truant, R., Zhang, S., Regier, J.L., Triezenberg, S.J., Reinberg, D., Flores, O., Ingles, C.J., et al. (1994). Binding of basal transcription factor TFIIH to the acidic activation domains of VP16 and p53. Mol Cell Biol 14, 7013-7024.

Yao, F., and Schaffer, P.A. (1995). An activity specified by the osteosarcoma line U2OS can substitute functionally for ICP0, a major regulatory protein of herpes simplex virus type 1. J Virol 69, 6249-6258.

Zhong, S., Müller, S., Ronchetti, S., Freemont, P.S., Dejean, A., and Pandolfi, P.P. (2000). Role of SUMO-1-modified PML in nuclear body formation. Blood 95, 2748-2752. 